\title{
Insights from the genome of a high alkaline cellulase producing Aspergillus fumigatus strain obtained from Peruvian Amazon rainforest
}

Sujay Paul ${ }^{1}$, Angel Zhang ${ }^{1}$, Yvette Ludeña ${ }^{1}$, Gretty K. Villena ${ }^{1 *}$, Fengan Yu$^{2}$, David H. Sherman $^{2}$ and Marcel Gutiérrez-Correa ${ }^{1 *}$

${ }^{1}$ Laboratorio de Micología y Biotecnología, Universidad Nacional Agraria La Molina, Av. La Molina s/n, Lima 12, Peru

${ }^{2}$ Life Sciences Institute, University of Michigan, 210 Washtenaw Avenue | Ann Arbor, MI 48109-2216, U.S.A.

*Corresponding authors: Laboratorio de Micología y Biotecnología, Universidad Nacional Agraria La Molina, Av. La Molina s/n, Lima 12, Peru Tel: +51 16147800 Ext. 463.

Emails: gkvch@lamolina.edu.pe 


\begin{abstract}
Here, we report the complete genome sequence of a high alkaline cellulase producing Aspergillus fumigatus strain LMB-35Aa isolated from soil of Peruvian Amazon rainforest. The genome is $\sim 27.5 \mathrm{mb}$ in size, comprises of 228 scaffolds with an average GC content of 50\%, and is predicted to contain a total of 8,660 protein-coding genes. Of which, 6,156 are with known function; it codes for 607 putative CAZymes families potentially involved in carbohydrate metabolism. Several important cellulose degrading genes, such as endoglucanase A, endoglucanase B, endoglucanase D and beta-glucosidase, are also identified. The genome of $A$. fumigatus strain LMB-35Aa represents the first whole sequenced genome of non-clinical, high cellulase producing A. fumigatus strain isolated from forest soil.
\end{abstract}

\title{
Keywords
}

Aspergillus fumigatus; lignocellulolytic enzyme; whole genome sequence; Amazon rainforest; CAZymes

Alkaline cellulases have enormous biotechnological applications in various industries, e.g., textile, food, paper, detergents and biofuels (Bhat, 2000; Sukumaran et al., 2005; Shah, 2013; Sajith et al., 2016); however, their industrial uses have been hindered due to lack of microbial strains yielding high alkaline cellulase (Galbe and Zacchi 2002; Vega et al., 2012). Here we report a high alkaline cellulase producing Aspergillus fumigatus strain collected from soil of Peruvian Amazon rainforest and its genome information.

Soil samples were collected at three locations from ground soil of remote Tingo Maria forest (Peruvian Amazon) in Huanuco, Peru and a total of 41 lignocellulosic fungal strains were identified using CMC-agar diffusion test (Teather and Wood 1982; Vega et al., 2012) (Table S1). Most of the identified strains were found to be different species of the genus Aspergillus. The endoglucanase activity of selected strains was quantified at $\mathrm{pH}$ 4.8, 7.4, 8.4, 9.4, 10.4 and 11.4, respectively using the method reported by Ghose (1987). The A. fumigatus strain LMB-35Aa 
showed the highest activity (100\% activity at $\mathrm{pH} 9.4$ after 72 hours incubation) and stability as well under a wider range of $\mathrm{pH}$ (with an activity of $20 \%$ to 100\%) (Fig. S1). Based on CMC-agar diffusion test and endoglucanase production, strain LMB-35Aa was selected for further characterization.

Genomic DNA was extracted from the culture of A. fumigatus LMB-35Aa using the Wizard $^{\circledR}$ Genomic DNA Purification Kit following manufacturer's instructions (Promega). Illumina paired end technology was used to sequence the whole genome of A. fumigatus LMB-35Aa at the DNA Sequencing Core of University of Michigan (Michigan, U.S.A.). Briefly, DNA was fragmented, end repaired, A' tagged, ligated to adaptors, size selected, enriched and the resultant libraries were sequenced on the Illumina HiSeq 2500 platform, generating paired-end sequence data with $100 \mathrm{nt}$ reads at each end, as per recommended protocols from Illumina, Inc. The raw sequencing data were first quality-checked with FastQC 2.2 program (Andrews, 2010) and then processed by inhouse Perl script to remove adapters and low quality reads. De-novo assembly of Illumina paired end data was performed using SPAdes assembler 3.1 (Bankevich et al, 2012) and SSPACE program (Boetzer et al., 2011) respectively. The resulting scaffolds were then used for protein prediction using Augustus.2.5.5 (Stanke et al., 2004) and annotated using NCBI BLAST with the proteins of Aspergillus from Uniprot database. 18S sequence was predicted in $A$. fumigatus LMB-35Aa genome using RNAmmer 1.2 server and the sequences of closest organism were selected to construct phylogenetic trees using MEGA tool through the maximum likelihood method (Jukes-Cantor model). The tree with the highest log likelihood is shown. Simple Sequence Repeats (SSR's) in the genome were identified using MISA Perl script (http://pgrc.ipk-gatersleben.de/misa/). An ideogram (Fig. 1) was generated to visualize the genomic features of A. fumigatus LMB-35Aa genome using circos plot (http://circos.ca/). Genome comparison of A. fumigatus LMB-35Aa with other reported A. fumigatus genomes was performed elaborately in this study using BRIG v0.95 (Fig. 2A), Mauve 2.3.1 (Fig. 2B), and circos plots (Fig. S2 and S3). Gene Ontology (GO) analysis of A. fumigatus LMB-35Aa was performed using Blast2GO PRO and visualized by WEGO plot. Pathway analysis was done using KAAS (KEGG Automatic Annotation Server) (http://www.genome.jp/tools/kaas/) with $A$. fumigatus as reference. CAZyme proteins were classified using online tool dbCAN (http://csbl.bmb.uga.edu/dbCAN/) with default parameters and secondary metabolite production 
was explored using antiSMASH (antibiotics and Secondary Metabolite Analysis SHell) ver.3 pipeline (Weber et al., 2015).

18S sequence based phylogenetic analysis of strain LMB-35Aa confirmed its classification to $A$. fumigatus (Fig. 3). A total of 228 scaffolds $(\mathrm{G}+\mathrm{C}$ content $=50 \%)$ has been generated from the $27.5 \mathrm{mb}$ of assembled genome and the $\mathrm{N} 50$ was approximately $726 \mathrm{~kb}$. A total of 8,660 proteincoding genes was predicted in A. fumigatus LMB-35Aa strain using Aspergillus Uniprot Protein Database, among which 6,156 were with known function. RNA genes (272), genes with Pfam domain $(6,805)$, signal peptide $(786)$, and transmembrane helices $(1,859)$ were also detected in this study (Table 1). Simple sequence repeat (SSR) analysis of A. fumigatus LMB-35Aa was performed and a total of 2,718 SSRs as well as 95 SSR-containing sequences were identified (Table S2). Recently, a biomass degrading A. fumigatus strain Z5 was isolated from plant straw compost heaps of China and sequenced (Miao et al., 2015). In this study, to better understand the genome orientation of A. fumigatus LMB-35Aa, we performed a whole genome sequence homology analysis using A. fumigatus strain Z5 and A. fumigatus strain Af293, the model clinical strain. Although, all the three genomes differ from each other in terms of GC\% (Af293 $=49.82 \%, \mathrm{Z} 5=49.20 \%$ and $\mathrm{LMB}-35 \mathrm{Aa}=50.00 \%$ ) and number of protein coding genes $($ Af293 $=9630, Z 5=9540$ and LMB-35Aa=8660) the homology analysis showed high degree of expected homology among them. Nevertheless, some clear genomic signatures make LMB-35Aa different from the other two strains. (Fig. 2A, 2B, S2, S3; Table S3 and S4). For example, genes like rodlets layer/hydrophobin (rodA or B/hyp1), conidial melanin-DHN (pksP/alb1), catalases (Cat1 and Cat2), 1,3 beta glucan, alkaline serin proteases (Alp1), chitin synthetases (ChsG and ChsE), Nonribosomal peptide synthase (Pes1), regulator of secondary metabolism (LaeA), Lornithine N5-oxygenase (SidA), Rheb small monomeric GTPase (RhbA) and trehalosephosphate synthase/phosphatase complex subunit (Tps1) are the major virulence factors that make A. fumigatus Af293 a model pathogenic strain (Abad et al., 2010). A comparison between A. fumigatus Af293 and A. fumigatus LMB-35Aa showed that most of the aforementioned virulence genes found in A. fumigatus Af293 had a sequence similarity of $99 \%$ (E value=0) to those found in A. fumigatus LMB-35Aa strain. Similarly, comparison between A. fumigatus Z5 strain and A. fumigatus LMB-35Aa indicated that except for rodA/hyp1 (with a similarity of 98\%), all the aforementioned genes of A. fumigatus Z5 strain showed a sequence similarity of 
99\%-100\% (E value=0) with A. fumigatus LMB-35Aa genome. However, some important aforesaid virulence genes which are present in both Af293 and Z5 genomes, such as rodB (regulator of adhesion), LaeA (regulator of secondary metabolism), RhbA (GTPase, Ras-related protein) and Tps1 (trehalose biosynthesis gene) were found absent in A. fumigatus LMB-35Aa genome. This result indicates that A. fumigatus LMB-35Aa is probably a non pathogenic strain and genomically different from the other two A. fumigatus strains.

Gene Ontology (GO) analysis of A. fumigatus LMB-35Aa strain indicated that a total of 6,156 genes were annotated in various categories including 'Biological Process' (BP) (5,593), 'Cellular Component' (CC) (4,253), and 'Molecular Function' (MF) (5,058). In BP, MF and CC categories, the highest represented GO terms were "metabolic process", "catalytic activity" and "cell", respectively (Fig. 4). Enzymatic pathway analysis using KAAS server revealed that the highest numbers of genes in KEGG pathways are involved in carbohydrate metabolism $(>500)$, followed by amino acid metabolism (>400), translation ( 300) and lipid metabolism ( 250) (Fig. S4). Plant biomass is composed of carbohydrate polymers such as cellulose, hemicellulose and aromatic polymer lignin. Carbohydrate-active enzymes (CAZymes) produced by parasites play a key role in the degradation of plant biomass. Currently, CAZymes have been categorized into six functional classes: glycoside hydrolases (GHs), glycosyltransferases (GTs), polysaccharide lyases (PLs), carbohydrate esterases (CEs), auxiliary activities (AAs) and carbohydrate-binding modules (CBMs). In the present study, by analyzing the A. fumigatus LMB-35Aa genome, a total of 607 putative CAZymes families (269 GHs, 105 GTs, 105 CEs, 58 CBMs, 55 AAs and 15 PLs) potentially involved in carbohydrate metabolism were identified using dbCAN web resource (only those with an e-value lower than $10^{-5}$ were considered) (Fig. S5). A. fumigatus strain Af293 codes for 587 (269 GHs, 109 GTs, 75 CEs, 62 CBMs, 57 AAs and 15 PLs) and strain Z5 codes for 569 (272 GHs, 98 GTs, 69 CEs, 61 CBMs, 55 AAs and 14 PLs) putative CAZymes families, which again indicates the uniqness of the current strain. The whole genome sequence annotation of A. fumigatus LMB-35Aa also revealed the occurrence of several important genes involved in the metabolism of complex carbohydrates. The most important cellulose degrading genes found in this study are endoglucanase A, endoglucanase B, endoglucanase D and betaglucosidase; endo-1,4-beta-xylanase, which is responsible for degrading the linear polysaccharide beta-1,4-xylan into xylose and thus breaking down hemicelluloses (one of the 
major components of plant cell wall), was also identified. Some important genes that are potentially involved in lactose/galactose metabolism such as lactose permease, galactose oxidase, galactose dehydrogenase, UDP-galactose-4-epimerase, galactose isomerase, etc., were detected. Other important genes that have a role in pectin metabolism, such as pectin lyase and pectinesterase, were also found. In fungal genomes, several genes have been found to form tightly linked clusters on the chromosome (Wiemann et al., 2013) and most of them produce pharmacologically important secondary metabolites (SM). A total of 31 SM gene clusters were detected in A. fumigatus LMB-35Aa using AntiSmash 3.0 online tool. Among all the clusters, nonribosomal peptide synthases (NRPS), polyketide synthases (PKS), and other groups were found to be the most abundant ones ( 7 clusters each), followed by terpene ( 5 clusters).

This is the first report on the whole genome sequence of a naturally occurring and high cellulase yielding A. fumigatus strain isolated from the soil of Amazon rainforest. This strain exhibited not only $100 \%$ activity at a certain point of alkaline $\mathrm{pH}(\mathrm{pH} 9.4)$ but also exhibited consistent high activity under a wide range of $\mathrm{pH}$, which may be highly beneficial in future industrial applications. We expect that this report of high-quality genome of A. fumigatus strain LMB35Aa will shed new light on the role of fungal genes for the production of biotechnologically important enzymes.

\section{Data Deposition}

The genome version discussed in this paper is from Aspergillus fumigatus strain LMB-35Aa. This Whole Genome Shotgun project has been deposited at DDBJ/ENA/GenBank under the accession MCQI00000000. The version described in this paper is version MCQI02000000 (MCQI00000000.2).

\section{Competing Interests}

The authors declare that they have no competing interests.

\section{Acknowledgements}

This work was supported by grants from Concejo Nacional de Ciencia y Tecnología of Peru [No 002-2013-CONCYTEC-FONDECYT and $\mathrm{N}^{\circ}$ 181-2015-FONDECYT-DE] and Programa 
Nacional de Innovación para la Competitividad y Productividad, Ministry of Production of Peru [No 176-FINCyT-IB-2013 and $\mathrm{N}^{\circ}$ 177-FINCyT-IB-2013]. This work was also supported by the National Science Foundation under the CCI Center for Selective C-H Functionalization (CHE1205646), and the Hans W. Vahlteich Professorship (D.H.S.). We acknowledge Genotypic Technology Pvt. Ltd and Eurofins Genomics India Pvt. Ltd, Bengaluru, India for additional analysis.

\section{References}

Abad, A., Fernández-Molina, J.V., Bikandi, J., Ramírez, A., Margareto, J., Sendino, J., Hernando, F.L., Pontón, J., Garaizar, J., Rementeria, A., 2010. What makes Aspergillus fumigatus a successful pathogen? Genes and molecules involved in invasive aspergillosis. Rev. Iberoam. Micol. 27(4), 155-182. doi:10.1016/j.riam.2010.10.003

Andrews, S., 2010. FastQC: A quality control tool for high throughput sequence data. http://www.bioinformatics.babraham.ac.uk/projects/fastqc/.

Bankevich, A., Nurk, S., Antipov, D., Gurevich, A. a., Dvorkin, M., Kulikov, A.S., Lesin, V.M., Nikolenko, S.I., Pham, S., Prjibelski, A.D., Pyshkin, A. V., Sirotkin, A. V., Vyahhi, N., Tesler, G., Alekseyev, M. a., Pevzner, P. a., 2012. SPAdes: A New Genome Assembly Algorithm and Its Applications to Single-Cell Sequencing. J. Comput. Biol. 19, 455-477. doi: $10.1089 / \mathrm{cmb} .2012 .0021$

Bhat, M.K., 2000. Cellulases and related enzymes in biotechnology. Biotechnol. Adv. doi:10.1016/S0734-9750(00)00041-0

Boetzer, M., Henkel, C. V., Jansen, H.J., Butler, D., Pirovano, W., 2011. Scaffolding preassembled contigs using SSPACE. Bioinformatics 27, 578-579. doi:10.1093/bioinformatics/btq683

Caffall, K.H., Mohnen, D., 2009. The structure, function, and biosynthesis of plant cell wall pectic polysaccharides. Carbohydr. Res. 344, 1879-1900. doi:10.1016/j.carres.2009.05.021

Galbe, M., Zacchi, G., 2002. A review of the production of ethanol from softwood. Appl. Microbiol. Biotechnol. doi:10.1007/s00253-002-1058-9

Huber, G.W., Iborra, S., Corma, A., 2006. Synthesis of transportation fuels from biomass: Chemistry, catalysts, and engineering. Chem. Rev. doi:10.1021/cr068360d 
Miao, Y., Liu, D., Li, G., Li, P., Xu, Y., Shen, Q., Zhang, R., 2015. Genome-wide transcriptomic analysis of a superior biomass-degrading strain of A. fumigatus revealed active lignocellulose-degrading genes. BMC Genomics 16, 459. doi:10.1186/s12864-015-1658-2

Miller, G.L., 1959. Use of Dinitrosalicylic Acid Reagent for Determination of Reducing Sugar. Anal. Chem. 31, 426-428. doi:10.1021/ac60147a030

Stanke, M., Steinkamp, R., Waack, S., Morgenstern, B., 2004. AUGUSTUS: A web server for gene finding in eukaryotes. Nucleic Acids Res. 32. doi:10.1093/nar/gkh379

Sukumaran, R.K., Singhania, R.R., Pandey, A., 2005. Microbial cellulases - Production, applications and challenges. J. Sci. Ind. Res. (India).

Teather, R.M., Wood, P.J., 1982. Use of Congo red-polysaccharide interactions in enumeration and characterization of cellulolytic bacteria from the bovine rumen. Appl. Environ. Microbiol. 43, 777-780.

Vega, K., Villena, G.K., Sarmiento, V.H., Ludeña, Y., Vera, N., Gutiérrez-Correa, M., 2012. Production of alkaline cellulase by fungi isolated from an undisturbed rain forest of peru. Biotechnol. Res. Int. 2012, 934325.

Weber, T., Blin, K., Duddela, S., Krug, D., Kim, H.U., Bruccoleri, R., Lee, S.Y., Fischbach, M.A., Müller, R., Wohlleben, W., Breitling, R., Takano, E., Medema, M.H., 2015. antiSMASH 3.0-a comprehensive resource for the genome mining of biosynthetic gene clusters. Nucleic Acids Res. 43, W237-43. doi:10.1093/nar/gkv437

Wiemann, P., Sieber, C.M.K., von Bargen, K.W., Studt, L., Niehaus, E.M., Espino, J.J., Huß, K., Michielse, C.B., Albermann, S., Wagner, D., Bergner, S. V., Connolly, L.R., Fischer, A., Reuter, G., Kleigrewe, K., Bald, T., Wingfield, B.D., Ophir, R., Freeman, S., Hippler, M., Smith, K.M., Brown, D.W., Proctor, R.H., Münsterkötter, M., Freitag, M., Humpf, H.U., Güldener, U., Tudzynski, B., 2013. Deciphering the Cryptic Genome: Genome-wide Analyses of the Rice Pathogen Fusarium fujikuroi Reveal Complex Regulation of Secondary Metabolism and Novel Metabolites. PLoS Pathog. 9. doi:10.1371/journal.ppat.1003475

\section{Figure legends}

\section{Fig 1.}

Circular representation of A. fumigatus LMB-35Aa genome showing genomic features. Track A: GC skew across the genome; Track B: Gene density across the genome, (red: minimum gene density; blue: maximum gene density); Track C: All the scaffolds of the genome. 


\section{Fig 2.}

A. Whole genome comparative analysis (BRIG) of A. fumigatus LMB-35Aa with the model clinical strain A. fumigatus Af293 and non clinical, cellulase prducing strain A. fumigatus Z5. The outermost dark brown circle represents the reference genome of A. fumigatus Af293; the next blue circle represents the genome of A. fumigatus Z5 strain and the innermost green circle represents A. fumigatus LMB-35Aa genome. The black line lying in between the A. fumigatus LMB-35Aa and genome-scale indicates the GC content.

B. Comparison of A. fumigatus LMB-35Aa and A. fumigatus Z5 genomes against the reference genome of A. fumigatus Af293 using Mauve tool. Coloured block outlines are known as Locally Collinear Blocks (LCBs) and are connected by corresponding coloured lines. LCBs represent the regions of similarity among the genomes that are homologous and have not undergone any rearrangement. Blocks lying above the center black horizontal line are in forward orientation while those below the center line indicate regions that are in the reverse orientation (inverted). Regions outside the blocks (white regions) show no homology among the genomes.

Fig 3. Phylogenetic tree showing the close relationship of sample A. fumigatus LMB-35A (18S Sequence scaffold), based on aligned sequences of 18S rRNA gene. Phylogenetic analysis was performed using MEGA. The tree was built using the maximum likelihood method. Bootstrap analysis (1000 replicates) was performed to validate the nodes.

Fig 4. WEGO plot visualization of GO terms identified in A. fumigatus LMB-35A. 
Table 1. General Features of A. fumigatus LMB-35A genome

\begin{tabular}{|l|l|}
\hline Total number of raw reads (million) & 27.59 \\
\hline Total number of processed reads (million) & 26.59 \\
\hline Genome size (bp) & $27,522,527$ \\
\hline G + C (\%) & 50 \\
\hline Genome coverage & $100 \mathrm{X}$ \\
\hline Scaffolds generated & 228 \\
\hline Total genes & 8,932 \\
\hline Total Protein coding genes & 8,660 \\
\hline Total genes with function prediction & 6,156 \\
\hline RNA genes & 272 \\
\hline Genes with Pfam domain & 6,805 \\
\hline Genes with signal peptide & 786 \\
\hline Genes with transmembrane helices & 1,859 \\
\hline Genes assigned to COG & 6,116 \\
\hline Scaffold N50 value (bp) & $7,26,133$ \\
\hline Scaffold L50 value & 12 \\
\hline
\end{tabular}




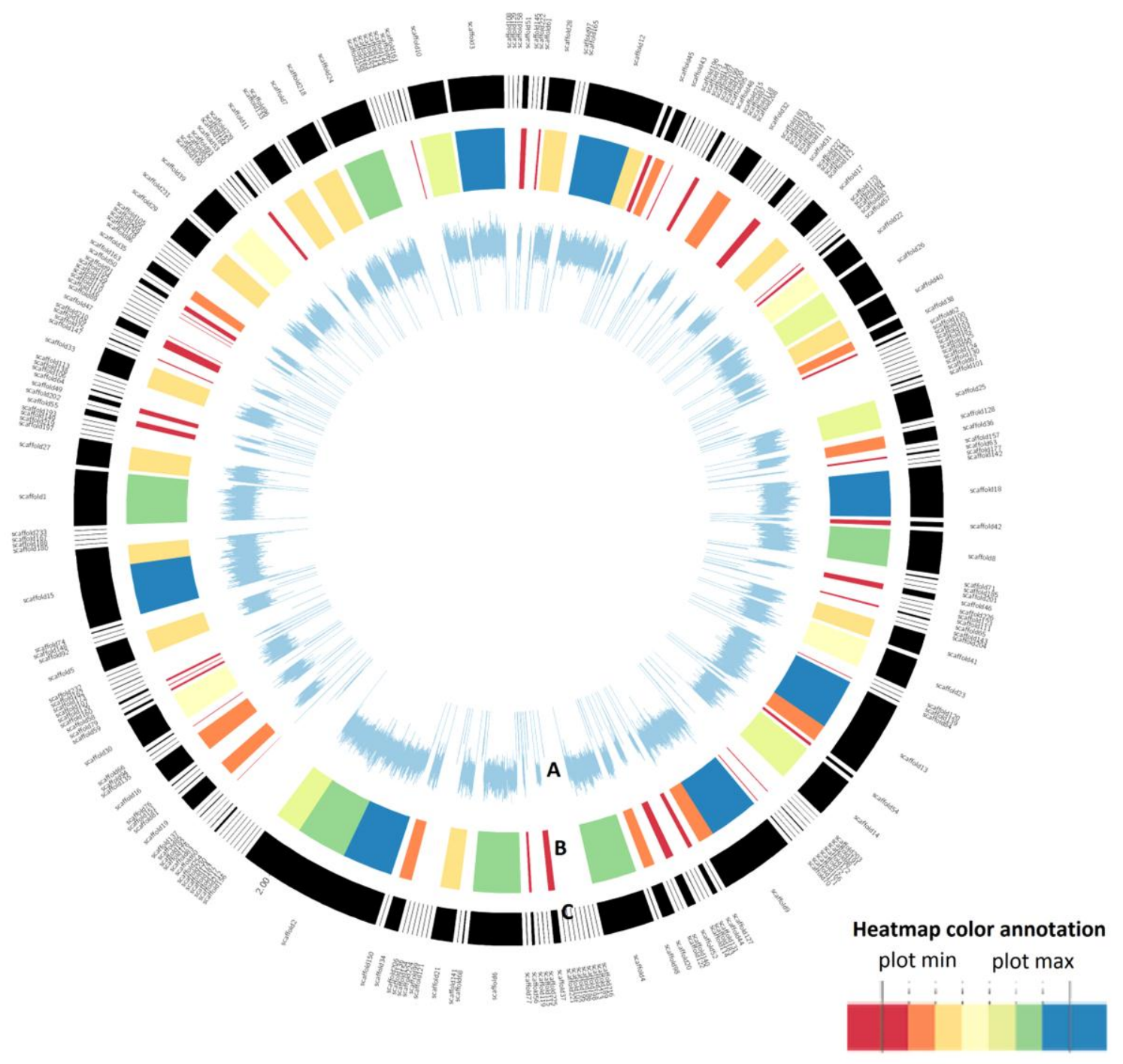

A: GC Skew

B: Gene Density

C: Genome Sequences (Scaffolds) 

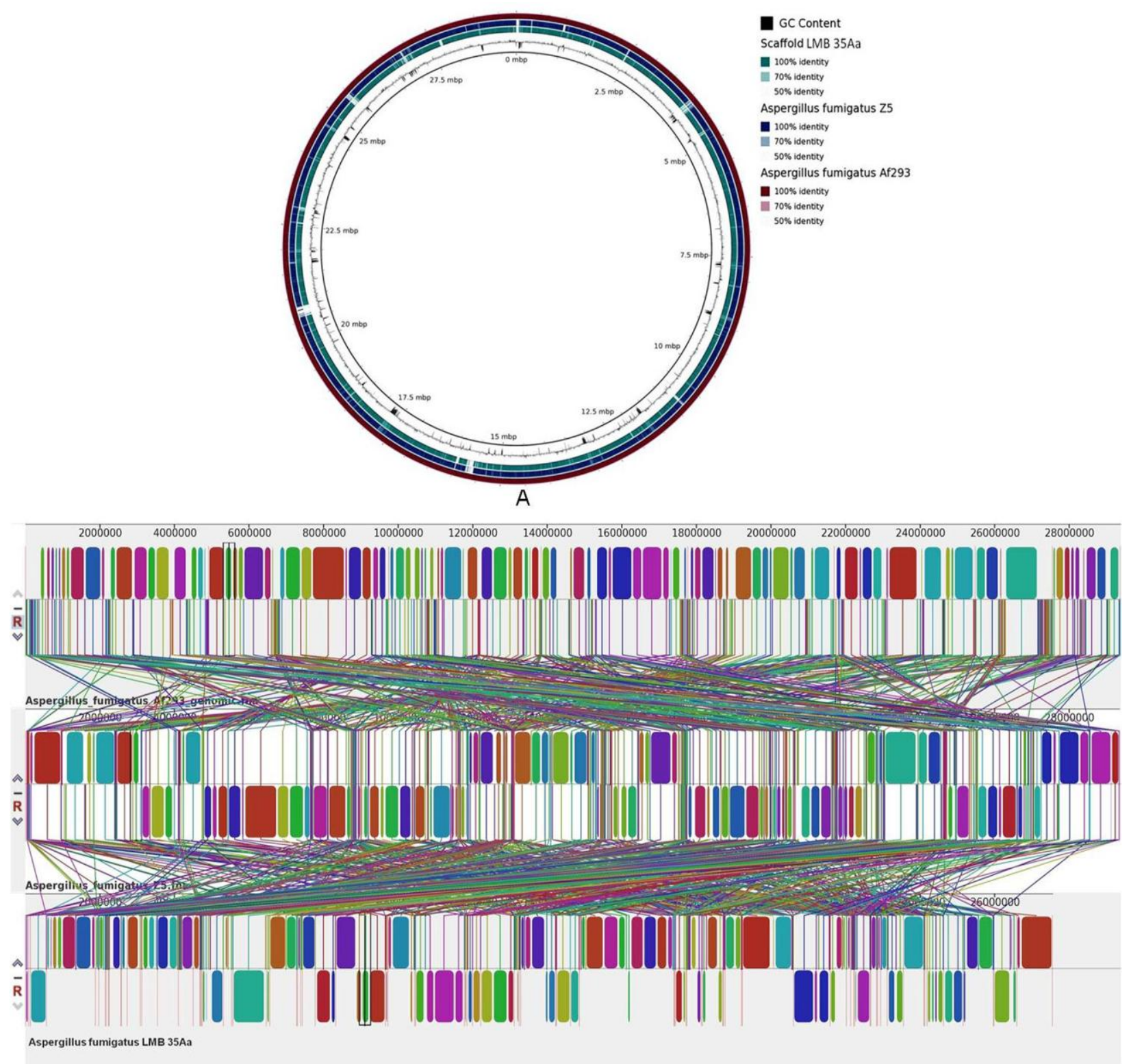


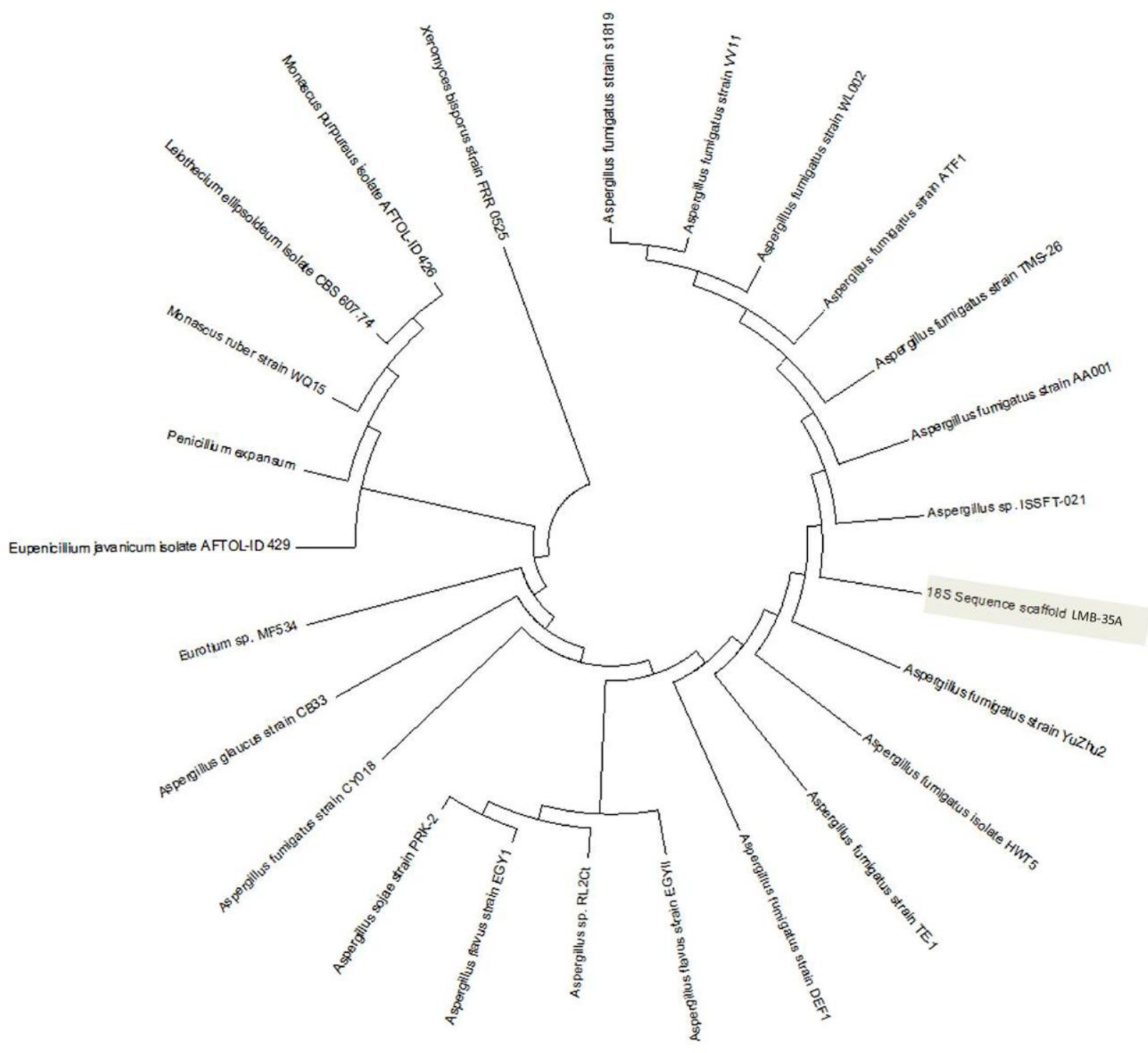




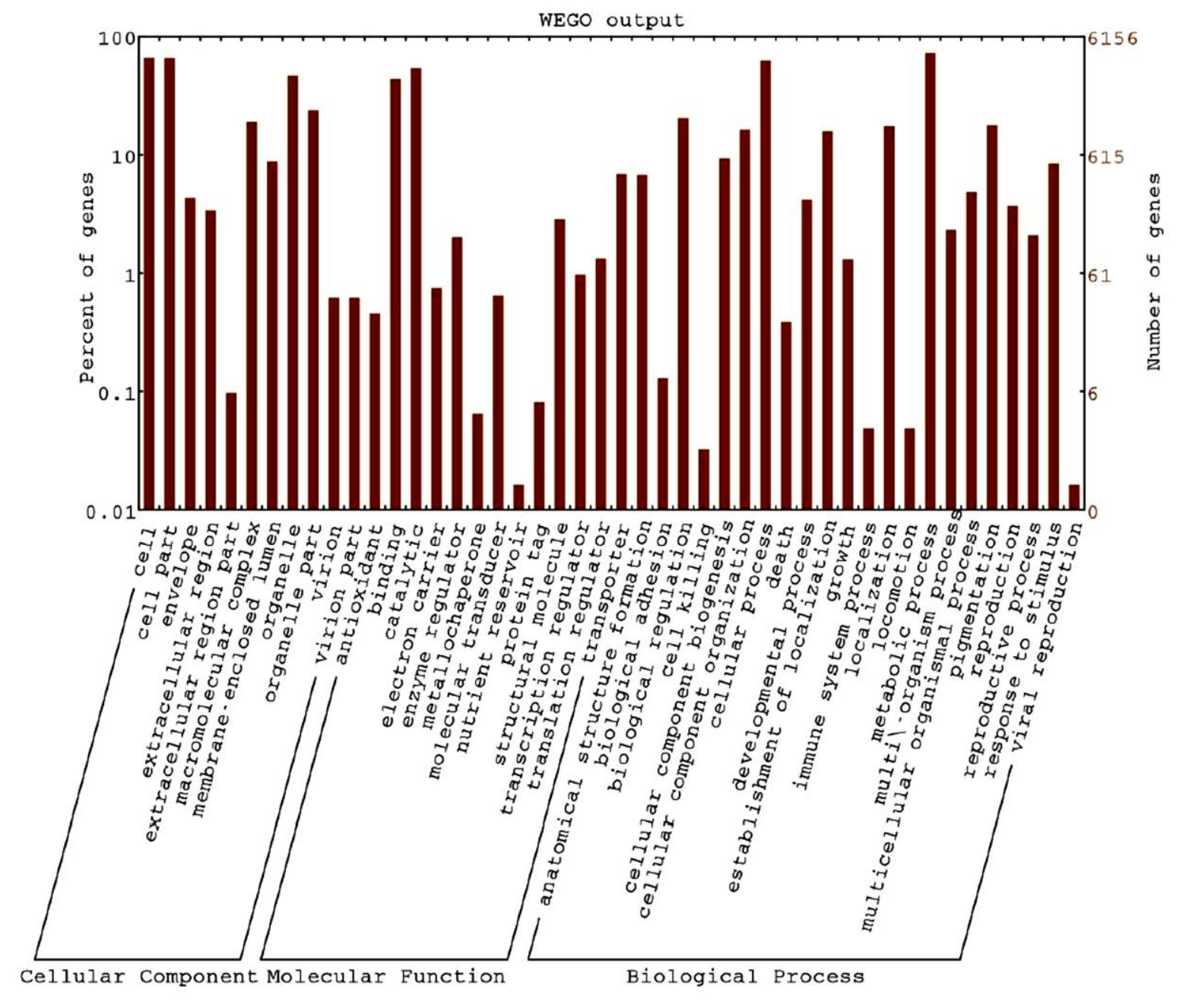

